\title{
Ranking Novel Regulatory Genes in Gene Expression Profiles using NetExpress*
}

\author{
Belma Yelbay \\ Department of Industrial \\ Engineering \\ Sabanci University, Turkey \\ byelbay@sabanciuniv.edu
}

\author{
Alexander Gow \\ Center for Molecular Medicine \\ and Genetics \\ Wayne State University, USA \\ agow@med.wayne.edu
}

\author{
Hasan M Jamil \\ Department of Computer \\ Science \\ University of Idaho, USA \\ jamil@uidaho.edu
}

\begin{abstract}
Understanding gene regulation by identifying gene products and determining their roles in regulatory networks is a complex process. A common computational method is to reverse engineer a regulatory network from gene expression profile, and sanitize the network using known information about the genes, their interactions and other properties to filter out unlikely interactors. Unfortunately, due to limited resources most gene expression studies have a limited and small number of time points, and most reverse engineering tools are unable to handle large numbers of genes. Both of these factors play significant roles in influencing the accuracy of the process. In this paper, we present a new gene ranking algorithm from gene expression profiles with a small number of time points so that the most relevant genes can be selected for reverse engineering. We also present a graphical interface called NetExpress, which adopts this algorithm and allows users to set control parameters to effect the desired outcome, and visualize the analysis for iterative fine tuning.
\end{abstract}

\section{CCS Concepts}

-Applied computing $\rightarrow$ Bioinformatics; Recognition of genes and regulatory elements; Biological networks; Computational genomics;

\section{INTRODUCTION}

Computational analysis of gene expression data can be a powerful approach for revealing the underlying regulatory patterns of regulatory networks and unraveling the mysteries of biological systems. Cell cultures principally can be subjected to targeted excitation in vitro to cause genes to initiate a signaling cascade among a set of gene products that potentially are part of a biological system or a network. These cascades manifest themselves as observable chemical activa-

\footnotetext{
${ }^{*}$ Research supported by National Science Foundation grants IIS 0612203 and DRL 1515550, and by National Institutes of Health grants NINDS NS056157 and NMSS RG4078.
}

Permission to make digital or hard copies of all or part of this work for personal or classroom use is granted without fee provided that copies are not made or distributed for profit or commercial advantage and that copies bear this notice and the full citation on the first page. Copyrights for components of this work owned by others than the author(s) must be honored. Abstracting with credit is permitted. To copy otherwise, or republish, to post on servers or to redistribute to lists, requires prior specific permission and/or a fee. Request permissions from permissions@acm.org.

SAC 2017, April 03 - 07, 2017, Marrakech, Morocco

(C) 2017 Copyright held by the owner/author(s). Publication rights licensed to ACM. ISBN 978-1-4503-4486-9/17/04 . .\$15.00

DOI: http://dx.doi.org/10.1145/3019612.3021289 tions and inhibitions (or deactivations), generally known as gene expression, that experiments aim to capture. The goal is to observe these cascades at sufficient temporal resolution so that no interactions are missed, and the complete gene expression time course or profile, is captured.

Under optimal conditions, the chain of activations and inhibitions captured in the time course can be translated into a processive network in which elements that are activated or deactivated at a given time point will follow elements that were active at a preceding time point, thereby giving rise to the concept of temporal regulation. For a small number of elements, deciphering such relationships is often not computationally difficult, but several factors render such an endeavor extremely complex and error prone.

For example, the large number of genes and other active gene products such as transcription factors and small RNAs give rise to a combinatorial explosion of possible interactions. If the number of time points in the experiment is large, such choices are further compounded. Additionally, the nature of the experimental design also introduces possible false positives and negatives, along with cross-talk among elements in different biological systems. These factors often contribute to the intractability of many computational models used to reverse engineer gene regulatory networks from gene expression time courses.

To combat the high computational cost, a very small gene subset is typically chosen from the time course that is considered most promising and relevant for reverse engineering. The number of time points is also usually very small to reduce chip hybridization cost. However, the smaller the number of time points, the coarser or more incomplete is the information content of the time course, and the more sophisticated inference mechanism is warranted. Thus, for a given computational model, the number of input genes becomes the most critical factor for ensuring the quality of inference because all other variables are already chosen.

In this paper, our goal is to present a toolkit, called NetExpress, that can be used to identify and extract the set of most promising $k$ genes from a gene expression time course. NetExpress is designed to support multiple data formats such as Affymetrix, Agilent and Illumina although the current version is equipped only with Agilent functionality. The simple user interface only requires setting an expression threshold, in the form of fold change per unit time, to distinguish true expression changes from background noise. The output is a set of potentially co-regulated promising genes, which reduces the size $k$ of the set of genes extracted where the higher the threshold, the smaller is the size $k$. 


\subsection{A Motivating Example}

NetExpress is motivated by a neurological study to determine the role of p53 in oligodendrocyte apoptosis. Neurodegeneration is linked to a number of diseases including multiple sclerosis, leukodystrophies, Alzheimer and Parkinson diseases [9]. Mutant gene expression in oligodendrocytes is also hypothesized to be responsible for protein misfolding and retention in the endoplasmic reticulum (ER) leading to hypomyelination via the unfolded protein response (UPR) [8]. One arm of this metabolic stress response, known as the PERK pathway, plays a significant role in the attenuation of global protein translation cell cycle arrest.

Recent studies in

our laboratory on rumpshaker (rsh) and myelin synthesis deficiency (msd) mutant mice suggest that crosstalk between the PERK and p53 pathways may be key to deciphering the mechanism of oligodendrocyte cell death [14]. This possibility is plausible because p53 is known to be involved in cell cycle progression check point at the G1-S boundary, and the interaction between ATF4 and p53 via ATF3 in the PERK pathway. However, initial laboratory studies cast doubt on the involvement of p53 in oligodendrocyte death under UPR conditions.

This observation is interesting because it raises the possibility of novel pathways in oligodendrocytes that are influenced by p53. Accordingly, the goal of the current study is to develop a computational approach to define the role of p53 and investigate its contribution to the UPR signaling cascade. To accomplish this task, we have identified genes from a UPR time course study in cultured cells that are either coordinately regulated with known UPR genes (Figure 1) or are targets of active elements in the PERK pathway, to construct a more extensive pathway that accounts for oligodendrocyte behavior in vivo and with high enough confidence so that novel interactors may be identified and their functions tested in vitro.

\subsection{Related Research}

Identifying co-expressed and co-regulated genes in gene expression time courses is an active area of research with a rich set of methods readily available. However, their biological relevance, overall effectiveness and generality still remain under intense scrutiny. Therefore it is difficult to pick one with confidence for ranking genes from an expression profile without carefully considering the method and the relevance to the analysis at hand.

The core of process of detecting relevant genes usually involves either the identification of co-expressing genes clusters, or the detection of differentially expressed genes. Efficient clustering methods have been applied in the past for the analysis of time course data (e.g., [12]), and some with specific attention to temporal expression relationship (e.g., [2]) for data from less complex experimental setup such as number of treatments, replicates and so on. Some of the other approaches aim to identify genes that vary in a statistically significant manner across various experimental conditions (e.g., [15]), and others try to use dimensionality reduction (e.g., [13]) to deal with a large number of variables.

Because most gene expression studies do not include more than 4-8 time points, as in our case, an analysis designed to function effectively with small numbers of time points is a prudent choice. After careful analysis and comparison of available methods we conclude that the Short Time-series Expression Miner (STEM) [1] algorithm proposed by Ernst and Bar-Joseph [7] is such a choice. Compared to the above approaches, STEM works for as small as three time points and works well with various experimental conditions. It also allows sophisticated parameter selection to fine tune the outcome. Although STEM ranks statistically significant clusters of expression profiles along with the genes that belong to those clusters, it does not extract individual genes of significance. In particular, STEM is not suitable for comparing multiple expression profiles directly in order to isolate genes that are potentially drivers of regulation such as the ones embedded in the knock-out expression profiles. In this paper, we adapt and extend STEM with this predictive capability to yield top $k$ genes by proposing the algorithm StemRank and incorporating it in an easy to use web based interface.

\section{STEMRANK ALGORITHM}

The StemRank algorithm aims to partition genes $g \in G$ into two sets - a set of genes $G^{+}$that are positively or negatively expressed under the experimental condition, and another set $G^{-}$that are not, and then rank the genes in both sets in order of significance. In our study, we have two sets of expression profiles, one from wild type cells in which p53 is present (labeled $\mathrm{p} 53^{+}$), and a mutant cell type in which p53 is deleted (labeled p53 ${ }^{-}$). We aim to identify the set of genes in $G^{+}$that are differently expressed in coordinated ways with p53 by comparing the expression patterns in both $\mathrm{p} 53^{+}$and $\mathrm{p} 53^{-}$. Intuitively, StemRank clusters expression patterns of the genes in both $\mathrm{p} 53^{+}$and $\mathrm{p} 53^{-}$, and then removes the genes that exhibit no change in their expression patterns from the genes that do. Finally, it ranks the genes in both groups in order of significance based on their strength of expression patterns, and selects the top $k$ genes.

\subsection{Expression Profile Clustering}

The number of expression profiles, called the candidate profiles $P$, that can be generated is given by the formula

$$
|P|=(2 c+1)^{n-1}
$$

where $n$ is the number of time points and $c$ is the possible number of absolute discrete expression levels. This is a quite large number of profiles and usually do not provide any useful information. So the trick is to collapse them into a much smaller set of model profiles such that the patterns in a model profile share expression traits. In particular, we would like to discretize expressions into three categories - into activation, inhibition or inert (no change). Then the possible number of model profiles for $n$ time points and three states is $m=3^{n-1}$. The collapse operation of the candidate profiles into $m$ model profiles then becomes an optimization 
problem of the form

$$
\max _{R \subset P,|R|=m}\left\{\min _{p_{1}, p_{2} \in R}\left\{d\left(p_{1}, p_{2}\right)\right\}\right\}
$$

where $d$ is a suitable distance function between profiles.

Once the model profiles are generated, genes $g \in G$ are uniquely assigned to each model profile based on the similarity of its expression pattern with the profile using the partial function $\alpha: G \leadsto R$, i.e., $\alpha(g)=p$. A model profile is identified as significant if the actual number of genes assigned is higher than the expected number of genes to be assigned to the model. The expected number of genes in each profile is computed by permuting the data points and making reassignment for the new sequence using the formula $\frac{\sum_{j} s_{p_{i}}^{j}}{n !}$, where $s_{p_{i}}^{j}$ is the number of genes assigned to the profile $p_{i}$ for the $j^{\text {th }}$ sequence. Finally, two significant profiles $p_{i}$ and $p_{j}$ are considered similar, and thus form a cluster, if $d\left(p_{i}, p_{j}\right) \leq \delta$, where $\delta=(1-\mu)$ is a threshold with a default value of 0.7 and $\mu$ is the minimum correlation between $p_{i}$ and $p_{j}$.

\subsection{Relevance Ranking}

Determination of gene relevance is based on the principle that a gene must be highly dependent on the environmental condition, i.e., the presence or absence of gene p53. In order to rank relevance, we first partition $G$ into $G^{+}$and $G^{-}$as the sets $\left\{g \mid \forall g\left(g \in G \wedge g_{1} \in p 53^{+} \wedge g_{2} \in p 53^{-} \wedge \alpha\left(g_{1}\right)=\right.\right.$ $\left.\left.p_{1} \wedge \alpha\left(g_{2}\right)=p_{2} \wedge g=g_{1}=g_{2} \Rightarrow p_{1} \neq p_{2}\right)\right\}$ and $G \backslash G^{+}$ respectively. The next and final step is to rank the genes in sets $G^{+}$and $G^{-}$in order of their promise. StemRank supports two choices: rank only the genes in significant profiles, or rank them all. The ranking function identifies genes as more promising if its absolute magnitude of expression is higher at any period than another gene in the same set. Therefore, ranking score of a gene $g \in G^{+}$or $g \in G^{-}$is given respectively by $\min _{g \in G^{+}}\left\{S_{g}\right\}$ and $\max _{g \in G^{-}}\left\{S_{g}\right\}$ where

$$
S_{g}=\bigcup_{g \in G, j=1}^{n-1}\left\{\left|e_{g, t_{2}}^{j}-e_{g, t_{1}}^{j}\right|\right\}
$$

and

$$
e_{g, t}^{j}=\frac{v_{g, t}^{j+1}-v_{g, t}^{j}}{v_{g, t}^{j}}
$$

where $t \in\left\{p 53^{+}, p 53^{-}\right\}, t_{1}=p 53^{+}, t_{2}=p 53^{-}$, and $v_{g, t}^{j}$ and $e_{g, t}^{j}$ are the value of expression at time point $j$ and the fractional change in expression value of $g$ under the test condition $t$ respectively. Genes in $G^{-}$are ordered in increasing order of their scores, and the ones on $G^{+}$in the reverse order. We finally choose top $k$ genes from both sets to report.

\section{EXPERIMENTAL STUDY}

As mentioned earlier, our interest in the function of p53 arose from a previous study [14] in rsh mutant mice. The rsh mouse is an excellent model of Pelizaeus-Merzbacher disease (PMD) in humans and stems from genetic lesions in the X chromosome-linked PROTEOLIPID PROTEIN (PLP1) gene [10]. This disease is a rare leukodystrophy that stems from a failure of the myelin sheath to develop normally in white matter tracts of the central nervous system.
Our immediate interest is in the PERK signaling cascade, which regulates ribosome assembly in the cell. Under UPR conditions, the activation of PERK in the ER attenuates global protein translation; however, a subset of UPR transcription factors are selectively transcribed and translated in a linear series of steps before protein translation is reinitiated (Figure 1). Thus, the PERK pathway serves as a time delay circuit to allow sufficient time for the cell to resolve the metabolic stress. Failure of the cell in achieving this goal may activate apoptosis [9].

\subsection{Role of p53 in Oligodendrocyte Cell Death}

During an analysis of the PERK pathway in rsh mice, we explored possible mechanisms by which the expression of mutant PLP1 might cause oligodendrocytes to undergo cell death [14]. We determined that the tumor-suppressor transcription factor p53 was expressed by these cells and localized to the nucleus, which suggests it might play an important role in apoptosis during metabolic stress [14]. The function of p53 has been studied in oligodendrocyte lineage cells in a number of contexts including low grade oligodendrogliomas (reviewed in [4]), oxidative stress [3] and demyelinating lesions [11]. Of particular interest to the current study, the demonstration of p53-mediated oligodendrocyte death in cuprizone-demyelinated mice suggests that this transcription factor could regulate apoptosis in rsh mice [11].

However, we observed that p53+ rsh oligodendrocytes exhibit normal morphology and were not labeled by markers of apoptosis, suggesting that nuclear p53 does not drive cell death in these mutants and that this transcription factor might exhibit an alternate function(s) in the context of the UPR. We undertook the current study to characterize the p53 regulatory network under metabolic stress conditions with the aim to identify novel regulators in the PERK pathway that can be further studied in vitro for validation. It is thus critical that we identify only the most promising few genes to reduce experimental costs in time and real dollars.

\subsection{Cell Lines and Culture Conditions}

Both of the human cell lines used in the current study, the parent line HCT116 $\mathrm{p} 53^{+/+}$cells and the HCT116 p53-/derivative line, were kind gifts from Dr. Bert Vogelstein, Johns Hopkins University, Baltimore, MD [5]. These cells were cultured at $37^{\circ} \mathrm{C}$ and $5 \% \mathrm{CO}_{2}$ in McCoy's $5 \mathrm{~A}$ medium (Gibco, Life Technologies, Grand Island, NY) supplemented with 10\% fetal bovine serum (Hyclone, Logan, UT), L-glutamine (Gibco) and penicilin/streptomycin (Gibco). The cultures were trypsinized using $0.25 \%$ trypsin-EDTA (Gibco) for $5 \mathrm{~min}$ at room temperature every three days and passaged to a seeding density of $10 \%$ in T75 flasks (ThermoFisher Scientific, Barrington, IL).

\subsection{RNA Purification}

Two days prior to experiment, cells in T75 flasks were trypsinized and used to seed $100 \mathrm{~mm}$ culture dishes at $20 \%$ confluence. The experiment was initiated by the addition of $2 \mathrm{mg} / \mathrm{ml}$ stock tunicamycin (Sigma, St Louis, MO) dissolved in dimethyl sulfoxide (Sigma) to the culture medium to a final concentration of $2 \mu \mathrm{g} / \mathrm{ml}$ (i.e. 1000x stock). Cells were exposed to tunicamycin for $0,2,4$ or 9 hours before they were rinsed twice with phosphate-buffered saline without calcium or magnesium (Gibco) and lysed in $3 \mathrm{ml}$ of 10 $\mathrm{mM}$ Tris $\mathrm{pH} 7$, containing $4 \mathrm{M}$ guanidinium isothiocyanate 
(Sigma), 0.14 M $\beta$-mercaptoethanol and $0.5 \%$ sodium lauryl sarcosinate (Sigma) as previously described [6]. Total RNA was purified over $1.7 \mathrm{ml}$ gradients of $5.7 \mathrm{M} \mathrm{CsCl}$ (Sigma) using an SW50.1 rotor in a Beckman L8 ultracentrifuge (Beckman Coulter, Brea, CA) at $22{ }^{\circ} \mathrm{C}$ for $16-18$ hours.

\section{ANALYSIS AND DISCUSSION}

The ranked output from NetExpress in this study considers only genes with specific functions, in our case transcription factors, which are further divided into two classes that are dependent or independent on p53. This novel approach reveals interesting features in the data that are important for our goals to determine if p53 is regulated by metabolic stress and if the p53 and UPR pathways interact.

First, analysis of the 12 most highly-ranked p53-independent genes includes the major transcription factors in the UPR (Figure 1), DDIT3, ATF3, XBP and CeBP-beta. Furthermore, transcription factors such as NFE2L2 - a central regulator of antioxidant pathways and is under investigation with pharmacological inhibitors for multiple sclerosis; NFIL3 regulates expansion of T-cells and may be important for activation of the adaptive immune system under metabolic stress conditions; and CREB3 - regulates T-cell migration and is necessary for enhanced protein degradation pathways during metabolic stress, are included in this group.

Second, although p53 expression is rapidly induced during the UPR with tunicamycin, expression of most of the UPR genes is independent of this master regulator of cell death. This is interesting because the UPR literature is heavily aligned with the notion that activation of the UPR is necessary for apoptosis of metabolically-stressed cells. The independence of UPR genes from p53 suggests that the 2 pathways can operate independently. Surprisingly, the master regulator of the UPR, ATF4, is revealed as a p53-dependent transcription factor in this analysis. However, ATF4 is ranked 394/436 p53-dependent genes which is only a marginal association with p53 at best. Together, this analysis indicates that the UPR and p53 pathways are parallel but independently regulated under metabolic stress conditions.

\section{CONCLUSIONS}

STEM has been successfully used in more than thirty research [1]) since its publication that required clustering of gene expression time courses, and to identify differentially expressed genes. Its diverse use, and its applicability to our study, amply demonstrates a need for a generalized toolbox based on STEM for profile clustering and gene selection. Our goal in this paper was to fill that need as part of our research in gene regulatory network prediction.

We have adapted STEM for yielding a list of ranked differentially expressed genes in order of significance. We also made it a part of a downstream reverse engineering system using which now regulatory network prediction and validation is possible in one single environment. Although the ranking procedure in the current version of NetExpress is simple and ranks genes based on the strength of expression, combined with STEM's ability to identify statistically significant profiles it helps rank them pretty well.

\section{ACKNOWLEDGEMENT}

The authors would like to thank Aminul Islam for implementing the prototype NetExpress system.

\section{REFERENCES}

[1] STEM Home Page. http://www.cs.cmu.edu/ jernst/stem/.

[2] Z. Bar-Joseph, G. Gerber, I. Simon, D. K. Gifford, and T. S. Jaakkola. Comparing the continuous representation of time-series expression profiles to identify differentially expressed genes. PNAS, 100(18):10146-10151, Sept. 2003.

[3] P. Bonini, S. Cicconi, A. Cardinale, C. Vitale, A. L. Serafino, M. T. Ciotti, and L. N.-L. Marlier. Oxidative stress induces p53-mediated apoptosis in glia: p53 transcription-independent way to die. Journal of Neuroscience Research, 75(1):83-95, 2004.

[4] T. D. Bourne and D. Schiff. Update on molecular findings, management and outcome in low-grade gliomas. Nature Reviews Neurology, 6:695-701, dec 2010.

[5] F. Bunz, A. Dutriaux, C. Lengauer, T. Waldman, S. Zhou, J. P. Brown, J. M. Sedivy, K. W. Kinzler, and B. Vogelstein. Requirement for p53 and p21 to sustain g2 arrest after dna damage. Science, 282(5393):1497-1501, 1998.

[6] J. M. Chirgwin, A. E. Przybyla, R. J. MacDonald, and W. J. Rutter. Isolation of biologically active ribonucleic acid from sources enriched in ribonuclease. Biochemistry, 18(24):5294-5299, 1979.

[7] J. Ernst and Z. B. Joseph. STEM: a tool for the analysis of short time series gene expression data. BMC Bioinformatics, 7(1):191+, 2006.

[8] A. Gow. Protein misfolding as a disease determinant. In R. A. Lazzarini, editor, Myelin Biology and Disorders, volume 2, chapter 42, pages 1009-1036. Elsevier Science, 2004.

[9] A. Gow and L. Wrabetz. CHOP and the endoplasmic reticulum stress response in myelinating glia. Current Opinion in Neurobiology, 19(5):505-510, 2009.

[10] L. D. Hudson and N. L. Nadon. Amino acid substitutions in proteolipid protein that cause dysmyelination. In R. E. Martenson, editor, Myelin: Biology and Chemistry, pages 677-702. CRC Press, Boca Raton, 1992.

[11] J. Li, C. A. Ghiani, J. Y. Y. Kim, A. Liu, J. Sandoval, J. DeVellis, and P. Casaccia-Bonnefil. Inhibition of p53 transcriptional activity: a potential target for future development of therapeutic strategies for primary demyelination. The Journal of Neuroscience, 28(24):6118-6127, June 2008.

[12] A. V. Lukashin and R. Fuchs. Analysis of temporal gene expression profiles: clustering by simulated annealing and determining the optimal number of clusters. Bioinformatics, 17(5):405-414, 2001.

[13] S. Ma and M. R. Kosorok. Identification of differential gene pathways with principal component analysis. Bioinformatics, 25(7):882-889, Apr. 2009.

[14] R. Sharma, H. Jiang, L. Zhong, J. Tseng, and A. Gow. Minimal role for activating transcription factor 3 in the oligodendrocyte unfolded protein response in vivo. Neurochemistry, 102(5):1703-1712, Sept. 2007.

[15] A. Tanay, R. Sharan, and R. Shamir. Discovering statistically significant biclusters in gene expression data. Bioinformatics, 18(suppl 1):S136-S144, July 2002. 